Instrumental Achievements

\title{
Porous Membrane Phase Separator in Solvent Extraction
}

\author{
Kousaburo OHaShi*, Syozo KoKubo**, Shôhei TAMURA*** and Katsumi Yamamoto* \\ *Department of Chemistry, Faculty of Science, Ibaraki University, Mito 310 \\ **Kokubo Seiki K. K., 2, Tomioka, Koutou, Tokyo 135 \\ ***Hamamatsu Photonics K. K., 3, Toranomon, Tokyo 105
}

Recently, a solvent extraction-flow injection analysis(FIA) ${ }^{1-3}$ has been developed which is a useful technique for the pre-concentration of metal ions prior to the determination of metal ions contained in a small volume sample solution by atomic absorption spectrophotometry.

A porous membrane separator is used to develop the solvent extraction-FIA. Consequently, the solvent extraction-FIA is very effective for the determination of trace amounts of metal ions, even those which are contained in a large amount of sample solution.

In this work, the apparatus with a phase separator, shown in Fig. 1, was developed. It was applied to the solvent extraction separation and determination of $10^{-7}$ mol dm${ }^{-3}$ level copper(II) ion with 5-octyloxymethyl-8quinolinol. This liquid is characterized by high hydrophobicity and by high selectivity toward copper(II) $^{4}$ and molybdenum(VI) ${ }^{5}$ in atomic absorption

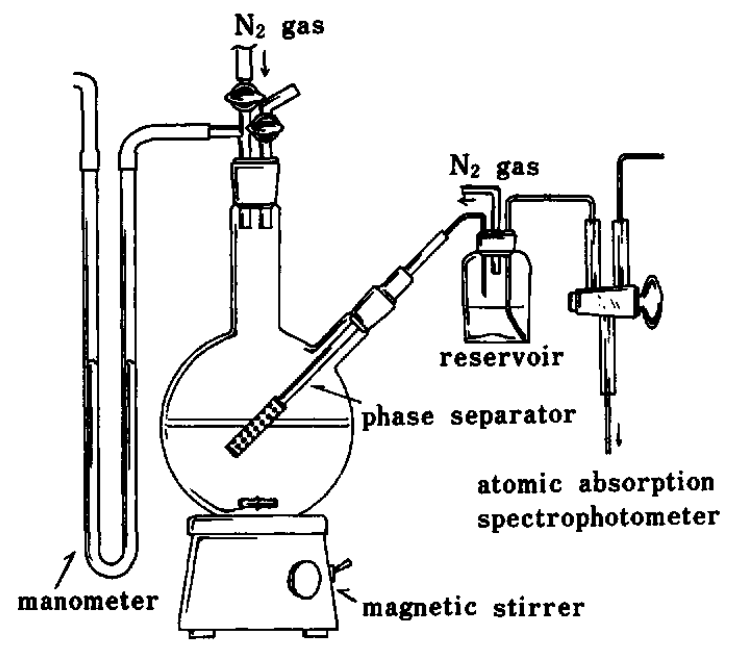

Fig. I Constructuion of the apparatus for the separation of metal ion.

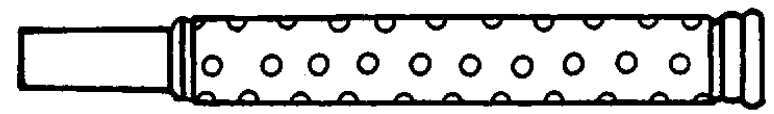

Fig. 2 Structure of porous membrane phase separator. spectrophotometry.

The apparatus comprises a flat-bottomed reaction flask $\left(1000 \mathrm{~cm}^{3}\right)$, a porous membrane phase separator (Kokubo Seiki, K. K., Fig. 2) (diameter, $10 \mathrm{~mm}$; length, $50 \mathrm{~mm}$ ), a manometer, and a magnetic stirrer. Nitrogen gas was passed through the flask to separate the organic phase from the aqueous phase by using the porous membrane phase separator while stirring. The pressure of nitrogen gas was adjusted at $30 \mathrm{mmHg}$. The organic phase comes out through a teflon tube (diameter, $2 \mathrm{~mm}$ ). The organic phase, once separated, was put into a reservoir, then introduced to the atomizer of the atomic absorption spectrophotometer. When the phase separator was put completely in the solution, the organic solvent came out together with the aqueous solution. Therefore, three-fourths of it, as shown in Fig. 1, was put in the solution. The separator, which was dried at $60^{\circ} \mathrm{C}$ for $4 \mathrm{~h}$ before use, was immersed in a DIBK solution of 5-octyloxymethyl-8-quinolinol.

The effects of the stirring time and of the concentration of 5-octyloxymethyl-8-quinolinol on the extraction of $6.00 \times 10^{-6} \mathrm{~mol} \mathrm{\textrm {dm } ^ { - 3 }}$ copper(II) with $5.00 \times 10^{-3} \mathrm{~mol} \mathrm{dm}^{-3}$ 5-octyloxymethyl-8-quinolinol at pH 3.0 were investigated for MIBK and DIBK. In the case of MIBK, the difference between the amounts of copper(II) ion found and calculated increased with the increment of the volume ratio of the aqueous phase to MIBK. This should be ascribed to the large solubility of MIBK in water. On the other hand, in the case of DIBK, reasonable results were obtained even for the volume ratio of aqueous phase to DIBK of $60: 1$. The extraction of a $10^{-7} \mathrm{~mol} \mathrm{dm}^{-3}$ level copper(II) with $1.00 \times 10^{-2} \mathrm{~mol} \mathrm{dm}^{-3} \quad 5$-octyloxymethyl-8-quinolinol reached equilibrium by being stirred for $20 \mathrm{~min}$ at $\mathrm{pH}$ 3.0.

Figure 3 represents the effect of $\mathrm{pH}$ on the extractability of copper(II) with 5-octyloxymethyl-8quinolinol into MIBK or DIBK. The complete extraction was achieved at $\mathrm{pH} 1.9$ and 2.1 for MIBK and DIBK, respectively.

The effect of diverse ions on the extractivedetermination of $5.54 \times 10^{-7} \mathrm{~mol} \mathrm{dm}^{-3}$ copper(II) with $1.00 \times 10^{-2} \mathrm{~mol} \mathrm{dm}^{-3} 5$-octyloxymethyl-8-quinolinol was studied at $\mathrm{pH} 2.8$ for the volume ratio of aqueous phase to DIBK of $60: 1$. At concentrations up to 


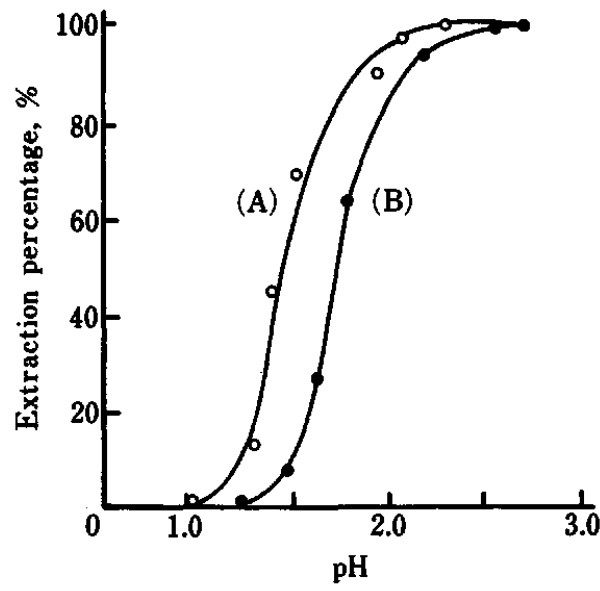

Fig. 3 Effect of $\mathrm{pH}$ on the extraction of copper(II) with 5-octyloxymethyl-8-quinolinol $\left(\mathrm{HO}_{8} \mathrm{Q}\right)$. [Cu(II)], 3.69 $\times 10^{-5}$ $\mathrm{mol} \mathrm{dm}-3$; [HO $\mathrm{HO}_{8}$ ], $1.00 \times 10^{-2} \mathrm{~mol} \mathrm{dm}^{-3}$. Organic solvent, (A) isobutyl methyl ketone, (B) diisobutyl ketone.

$1.00 \times 10^{-3} \mathrm{~mol} \mathrm{dm}^{-3}$ each, metal ions including thallium(I), cobalt(II), nickel(II), zinc(II), lead(II), cadmium(II) and chromium(III) did not interfere. In the presence of $1.00 \times 10^{-3} \mathrm{~mol} \mathrm{dm}^{-3}$ of iron(III), molybdenum(VI), tin(IV), tungsten(VI), zirconium(IV),
gallium(III) or vanadium(V), the determination of copper(II) was seriously interfered with.

The present apparatus will be applicable to the selective separation and determination of trace amounts of metal ions with highly selective extractant by utilizing the extraction and the subsequent backextraction procedures.

This work was partially supported by a Grant-in-Aid for Scientific Research from the Ministry of Education, Science and Culture.

\section{References}

1. J. A. Sweileh and F. F. Cantwell, Anal. Chem., 57, 420 (1985).

2. M. Gallego and M. Valcarcel, Anal. Chim. Acta, 169, 161 (1985).

3. T. Kumamaru, Y. Nitta, F. Nakata, H. Matsuo and M. Ikeda, Anal. Chim. Acta, 174, 183 (1985).

4. K. Ohashi, S. Nakata, M. Katsume, K. Nakamura and K. Yamamoto, Anal. Sci., 1, 467 (1985).

5. K. Ohashi, M. Inose, K. Nakamura and K. Yamamoto, Anal. Sci., 2, 439 (1986).

(Received July 31, 1987)

(Accepted September 16, 1987) 